\title{
WEAK LINE QUASARS AT HIGH REDSHIFT: EXTREMELY HIGH ACCRETION RATES OR ANEMIC BROAD-LINE REGIONS?
}

\author{
Ohad Shemmer ${ }^{1}$, Benny Trakhtenbrot ${ }^{2}$, Scott F. Anderson ${ }^{3}$, W. N. Brandt ${ }^{4,5}$, Aleksandar M. Diamond-Stanic ${ }^{6}$, \\ Xiaohui Fan ${ }^{6}$, Paulina Lira ${ }^{7}$, Hagai Netzer ${ }^{2}$, Richard M. Plotkin ${ }^{8}$, Gordon T. Richards ${ }^{9}$, Donald P. Schneider ${ }^{4}$, \\ and Michael A. Strauss ${ }^{10}$ \\ ${ }^{1}$ Department of Physics, University of North Texas, Denton, TX 76203, USA; ohad@unt.edu \\ ${ }^{2}$ School of Physics and Astronomy and the Wise Observatory, The Raymond and Beverly Sackler Faculty of Exact Sciences, \\ Tel-Aviv University, Tel-Aviv 69978, Israel \\ ${ }^{3}$ Department of Astronomy, University of Washington, Box 351580, Seattle, WA 98195, USA \\ ${ }^{4}$ Department of Astronomy and Astrophysics, The Pennsylvania State University, University Park, PA 16802, USA \\ ${ }^{5}$ Institute for Gravitation and the Cosmos, The Pennsylvania State University, University Park, PA 16802, USA \\ ${ }^{6}$ Steward Observatory, University of Arizona, 933 North Cherry Avenue, Tucson, AZ 85721, USA \\ ${ }^{7}$ Departamento de Astronomía, Universidad de Chile, Camino del Observatorio 1515, Santiago, Chile \\ ${ }^{8}$ Astronomical Institute "Anton Pannekoek," University of Amsterdam, Science Park 904, 1098 XH, Amsterdam, The Netherlands \\ ${ }^{9}$ Department of Physics, Drexel University, 3141 Chestnut Street, Philadelphia, PA 19104, USA \\ ${ }^{10}$ Princeton University Observatory, Peyton Hall, Princeton, NJ 08544, USA \\ Received 2010 August 13; accepted 2010 September 8; published 2010 September 28
}

\begin{abstract}
We present Gemini-North $K$-band spectra of two representative members of the class of high-redshift quasars with exceptionally weak rest-frame ultraviolet emission lines (WLQs), SDSS J114153.34+021924.3 at $z=3.55$ and SDSS J123743.08+630144.9 at $z=3.49$. In both sources, we detect an unusually weak broad $\mathrm{H} \beta$ line and place tight upper limits on the strengths of their [O III] lines. Virial, $\mathrm{H} \beta$-based black hole mass determinations indicate normalized accretion rates of $L / L_{\mathrm{Edd}}=0.4$ for these sources, which is well within the range observed for typical quasars with similar luminosities and redshifts. We also present high-quality XMM-Newton imaging spectroscopy of SDSS J114153.34+021924.3 and find a hard-X-ray photon index of $\Gamma=1.91_{-0.22}^{+0.24}$, which supports the virial $L / L$ Edd determination in this source. Our results suggest that the weakness of the broad emission lines in WLQs is not a consequence of an extreme continuum-emission source but instead due to abnormal broad emission line region properties.
\end{abstract}

Key words: galaxies: active - galaxies: nuclei - quasars: emission lines - quasars: individual (SDSS J114153.34+021924.3, SDSS J123743.08+630144.9) - X-rays: galaxies

Online-only material: color figure

\section{THE NATURE OF LINELESS QUASARS AT HIGH REDSHIFT}

The Sloan Digital Sky Survey (SDSS; York et al. 2000) has, so far, discovered $\sim 80$ sources at $2.2 \leqslant z \leqslant 5.9$ with $v L_{v}(2500 \AA) \gtrsim 10^{46} \mathrm{erg} \mathrm{s}^{-1}$ that have almost featureless restframe ultraviolet (UV) spectra (Fan et al. 1999; Anderson et al. 2001; Collinge et al. 2005; Schneider et al. 2005; DiamondStanic et al. 2009, hereafter DS09; Plotkin et al. 2010). By virtue of their largely featureless spectra, the redshifts of these sources can be determined reliably only from the onset of the Ly $\alpha$ forest or the presence of a Lyman limit system. Subsequent multiwavelength observations of several sources of this class have shown that they are unlikely to be highredshift galaxies with apparent quasar-like luminosities due to gravitational lensing amplification, dust-obscured quasars, or broad absorption line quasars (e.g., Shemmer et al. 2006; DS09). Furthermore, based on their relative radio and X-ray weakness, Shemmer et al. (2009, hereafter S09) have argued against the possibility that such sources may be the long-sought, highredshift BL Lacertae objects (e.g., Stocke \& Perrenod 1981; see also DS09). Instead, S09 suggested that these sources are unbeamed quasars with extreme properties.

High-redshift quasars with weak or undetectable UV emission lines (hereafter weak emission line quasars or WLQs) are defined as quasars having rest-frame equivalent widths (EWs) of $<15.4 \AA$ for the Ly $\alpha+\mathrm{N} v$ emission-line complex (DS09).
This EW threshold marks the $3 \sigma$ limit at the low-EW end for the Ly $\alpha+\mathrm{N}$ v EW distribution in a sample of $\sim 3000$ SDSS quasars at $z>3$ with no broad absorption lines. Instead of the $\sim 4$ quasars statistically expected to lie below this threshold (assuming that the EW distribution is lognormal), $56 \mathrm{WLQs}$ are included in that sample, and there is no corresponding excess of "strong-lined" quasars beyond the $3 \sigma$ limit at the high-EW end (DS09). The reason for this excess of quasars with extremely weak UV emission lines is not yet understood. Spectroscopic monitoring of four WLQs further suggests that it is unlikely that the weakness of their lines can be explained by microlensing that temporarily and preferentially amplifies the continuum relative to the broad emission lines (DS09). This result supports the idea that the UV emission lines in WLQs are intrinsically weak.

Intrinsically weak UV emission lines in quasars may be a consequence of a peculiar continuum-source spectral energy distribution (SED) that has a relative deficiency in high-energy photons. For example, a combination of large black hole mass $\left(M_{\mathrm{BH}}\right)$ and extremely high accretion rate may result in a relatively narrow, UV-peaked SED (e.g., Leighly et al. 2007a, 2007b; Vasudevan \& Fabian 2007). In such a scenario, prominent high-ionization emission lines, such as $\mathrm{CIV}$, are suppressed relative to low-ionization emission lines such as $\mathrm{H} \beta$, which are not affected to the same extent. The peculiar spectral properties of the nearby $(z=0.19)$, high accretion rate quasar PHL 1811 may be explained by this effect. This quasar exhibits $\mathrm{EW}(\mathrm{C}$ IV $)=6.6 \AA$ (within the range observed for WLQs; DS09), 


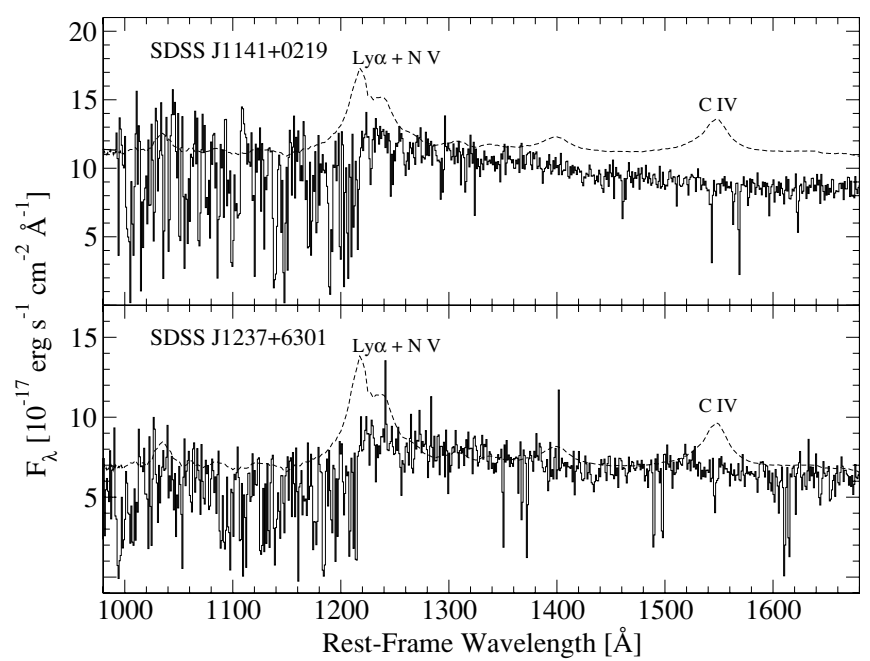

Figure 1. SDSS spectra of SDSS J1141+0219 (top) and SDSS J1237+6301 (bottom). Both spectra were resampled in bins of 1 A for clarity. The SDSS Early Data Release quasar spectrum template (Vanden Berk et al. 2001) is shown in each panel (dashed lines) for comparison; the SDSS quasar template was scaled arbitrarily in flux density, in each panel, and prominent emission lines are marked. Note the extreme weakness of the Ly $\alpha$ and C IV emission lines in each WLQ

while $\mathrm{H} \beta$, for example, has a more typical, but still relatively weak, quasar value of $\mathrm{EW}=50 \AA$ (Leighly et al. 2007a). At least one additional quasar at $z<2.2$, e.g., PG 1407+265 $(z=0.94$; McDowell et al. 1995), may also be similar to PHL 1811. Alternatively, WLQs may have abnormal broad emission line region (BELR) properties, such as a significant deficit of line-emitting gas in the BELR or a low BELR covering factor. In this scenario, their low-ionization emission lines are expected to be weak as well. It has also been suggested that the BELRs in WLQs may be in an early stage of formation (Hryniewicz et al. 2010).

In this work, we present the first near-infrared (NIR) spectroscopic observations of two representative WLQs, SDSS J114153.34+021924.3 at $z=3.55$ and SDSS J123743.08+ 630144.9 at $z=3.49$ (hereafter SDSS J1141+0219 and SDSS J1237+6301, respectively). Both sources were identified as candidate WLQs by Collinge et al. (2005). We also present a high-quality X-ray spectrum of SDSS J1141+0219, the first such of a WLQ. These observations were designed as a pilot study to (1) determine the normalized accretion rates (in terms of the Eddington ratio, $L_{\mathrm{bol}} / L_{\mathrm{Edd}}$, hereafter $\left.L / L_{\mathrm{Edd}}\right)$ in WLQs and test the hypothesis that extremely high accretion rates are responsible for the weakness of the UV emission lines, and (2) measure the properties of low-ionization emission lines in WLQs. We describe our observations and their results in Section 2, discuss their implications for BELRs in active galactic nuclei (AGNs) in Section 3, and provide a summary in Section 4. Throughout this Letter, luminosity distances are computed using the standard cosmological model $\left(H_{0}=70 \mathrm{~km} \mathrm{~s}^{-1} \mathrm{Mpc}^{-1}, \Omega_{\Lambda}=0.7\right.$, $\left.\Omega_{M}=0.3\right)$.

\section{OBSERVATIONS, DATA REDUCTION, AND RESULTS}

\subsection{NIR Spectroscopy}

SDSS J1141+0219 and SDSS J1237+6301 were the brightest WLQs with suitable redshifts for observing the $\mathrm{H} \beta$ spectral region in the center of the $K$ band. ${ }^{11}$ Their optical, X-ray, and

\footnotetext{
${ }^{11}$ Prior to the Plotkin et al. (2010) study, there were no suitable candidates for $H$-band spectroscopy, i.e., WLQs at $2.2 \lesssim z \lesssim 2.5$.
}

Table 1

Gemini-North NIRI $K$-band Observation Log

\begin{tabular}{cccccc}
\hline \hline & & \multicolumn{2}{c}{$\log \nu L_{v}$} & Exp. Time \\
Quasar & $z^{\mathrm{a}}$ & $z_{\text {sys }}^{\mathrm{b}}$ & $(2500 \AA)$ & Observation Dates & $(\mathrm{s})$ \\
\hline SDSS J1141+0219 & 3.48 & 3.55 & 46.7 & 2009 Dec 12, 13, 23 & 6325 \\
SDSS J1237+6301 & 3.42 & 3.49 & 46.5 & 2009 Dec 23, 24, 25 & 10865 \\
\hline
\end{tabular}

Notes.

a Redshift obtained from DS09, based on SDSS spectra.

b Systemic redshift (see Section 2.1 for details).

radio properties are typical of the WLQ population and, in particular, their $\mathrm{EW}(\mathrm{Ly} \alpha+\mathrm{N} \mathrm{v})$ are $3.3 \AA$ and $4.6 \AA$, respectively (DS09; S09). The SDSS spectra of these WLQs are shown in Figure 1. Spectroscopic observations were carried out at the Gemini-North Observatory using the Near InfraRed Imager and Spectrometer (NIRI; Hodapp et al. 2003) as program GN-2009A-Q-9. The observation log appears in Table 1. For both targets, we used the $f / 6$ camera with the 6 pixel centered slit $(0.75$ wide), the $K$ grism, and the broadband $K$ filter. The resulting spectral range and spectral resolution were $\Delta \lambda=1.90-2.49 \mu \mathrm{m}$ and $R=520$, respectively. Both targets were nodded along the slit to obtain optimal background subtraction. Exposure times for each subintegration were $275 \mathrm{~s}$ and $265 \mathrm{~s}$ and the total integration times were $6325 \mathrm{~s}$ and $10865 \mathrm{~s}$ for SDSS J1141+0219 and SDSS J1237+6301, respectively. We reduced the spectra using standard procedures of the IRAF ${ }^{12}$ Gemini package (version 1.8). Exposures from different nodding positions were used to subtract the sky emission and were then co-added. Wavelength calibration employed Ar exposures. Relative flux calibration was obtained by taking spectra of telluric standard stars (A0 V type) immediately before or after the science exposures. To compensate for possible slit losses in the flux calibration, we compared the total fluxes of our targets to those of nearby Two Micron All Sky Survey (2MASS; Skrutskie et al. 2006) point sources, imaged in the NIRI $2^{\prime} \times 2^{\prime}$ field of view. The uncertainty in the absolute flux calibration is estimated as $\sim 10 \%$, which is the quoted uncertainty on the 2MASS magnitudes. The final NIR spectra appear in Figure 2. The useful range of observed wavelengths is $\sim 2.0-2.4 \mu \mathrm{m}$, corresponding to rest frame $\sim 4400-5400 \AA$.

We modeled the spectra following the methods of Shemmer et al. (2004). In short, our model consisted of a linear continuum, fitted between two narrow ( $\pm 20 \AA$ ) rest-frame bands centered on $4700 \AA$ and $5100 \AA$, a broadened Fe II emission template (Boroson \& Green 1992), a broad $\left(1200 \mathrm{~km} \mathrm{~s}^{-1} \leqslant\right.$ FWHM $\leqslant 15,000 \mathrm{~km} \mathrm{~s}^{-1}$ ) Gaussian profile representing $\mathrm{H} \beta$, and three narrow $\left(300 \mathrm{~km} \mathrm{~s}^{-1} \leqslant \mathrm{FWHM} \leqslant 1200 \mathrm{~km} \mathrm{~s}^{-1}\right)$ Gaussian profiles: one for the narrow $\mathrm{H} \beta$ component and two for [O III] $\lambda \lambda 44959,5007$. The widths of the narrow Gaussians were tied to the same value and the $[\mathrm{O}$ III] lines were constrained to have the theoretical ratio $I([\mathrm{O} \mathrm{III}] \lambda 5007) / I([\mathrm{O} \mathrm{III}] \lambda 4959)=$ 2.95. The Fe II emission template was broadened by convolving it with a Gaussian profile whose width was free to vary within the velocity range used for the broad $\mathrm{H} \beta$. Our fitting results are summarized in Table 2, where error bars are given at a $90 \%$ confidence level, and the best-fit models appear in Figure 2. Upper limits on $\mathrm{EW}\left(\left[\mathrm{O}_{\mathrm{III}}\right] \lambda 5007\right)$ were determined by assuming a Gaussian profile with FWHM $=1000 \mathrm{~km} \mathrm{~s}^{-1}$

\footnotetext{
12 Image Reduction and Analysis Facility (IRAF) is distributed by the National Optical Astronomy Observatory, which is operated by AURA, Inc., under cooperative agreement with the National Science Foundation.
} 
Table 2

Derived Optical Properties

\begin{tabular}{|c|c|c|c|c|c|c|c|c|}
\hline \multirow[b]{2}{*}{ Quasar } & \multirow[b]{2}{*}{$\operatorname{FWHM}(\mathrm{H} \beta)^{\mathrm{a}}$} & \multicolumn{3}{|c|}{ Emission-Line Rest-Frame EW $(\AA ̊)$} & \multirow[b]{2}{*}{$\alpha^{\mathrm{c}}$} & \multirow[b]{2}{*}{$f_{\lambda}^{\mathrm{d}}$} & \multirow[b]{2}{*}{$\log M_{\mathrm{BH}}$} & \multirow[b]{2}{*}{$L / L_{\mathrm{Edd}}$} \\
\hline & & $\mathrm{H} \beta \lambda 4861$ & {$[\mathrm{O}$ III $] \lambda 5007$} & Fe II $\lambda \lambda 4434-4684^{b}$ & & & & \\
\hline SDSS J1141+0219 & $5.9_{-1.1}^{+1.0}$ & $20_{-3}^{+4}$ & $\leqslant 6$ & $65_{-9}^{+10}$ & -2.15 & 1.07 & 9.5 & 0.4 \\
\hline SDSS J1237+6301 & $5.2_{-1.0}^{+1.5}$ & $35_{-5}^{+8}$ & $\leqslant 7$ & $100_{-13}^{+12}$ & -0.98 & 0.63 & 9.3 & 0.4 \\
\hline
\end{tabular}

Notes.

${ }^{a}$ In units of $1000 \mathrm{~km} \mathrm{~s}^{-1}$, corrected for instrumental resolution.

${ }^{\mathrm{b}}$ Broadened by a Gaussian profile having an FWHM of $2440 \mathrm{~km} \mathrm{~s}^{-1}$ and $1090 \mathrm{~km} \mathrm{~s}^{-1}$, corrected for instrumental resolution, for SDSS J1141+0219 and SDSS J1237+6301, respectively.

${ }^{c}$ Optical continuum slope $\left(f_{\lambda} \propto \lambda^{\alpha}\right)$ determined in the $4700-5100 \AA$ A rest-frame band.

${ }^{\mathrm{d}}$ Flux density at rest-frame $5100 \AA$ in units of $10^{-17} \mathrm{erg} \mathrm{cm}^{-2} \mathrm{~s}^{-1} \AA^{-1}$.

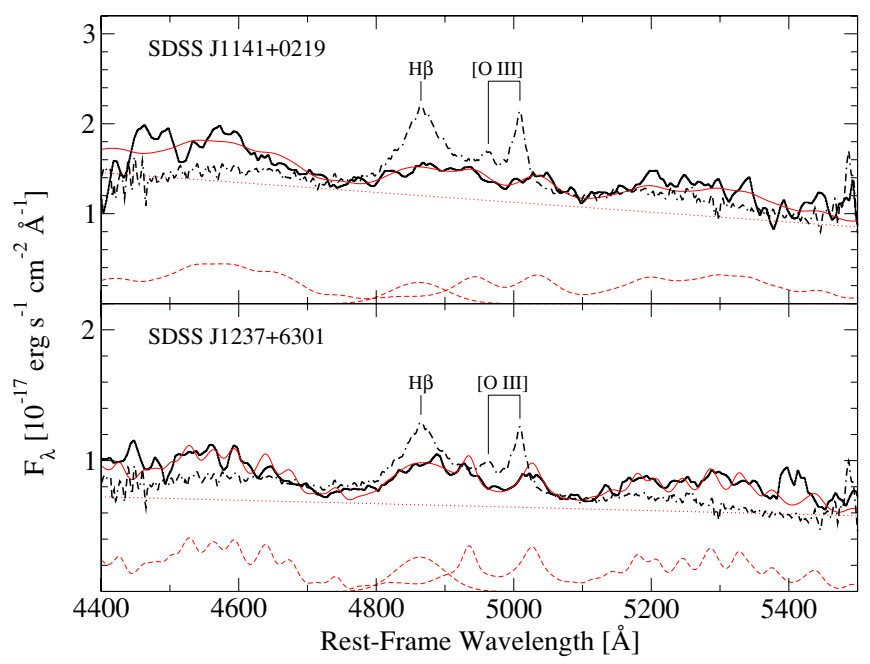

Figure 2. Gemini-North NIRI spectra of SDSS J1141+0219 (top) and SDSS J1237+6301 (bottom). Both spectra (thick solid line) were resampled in bins of $5 \AA$ for clarity. A mean spectrum of 33 quasars from the Shemmer et al. (2004) and Netzer et al. (2007) comparison sample is shown (dot-dashed thick lines) next to each WLQ spectrum for comparison; the mean spectrum of the comparison sample was scaled in flux to match the $5100 \AA$ flux density in each WLQ spectrum, and prominent emission lines are marked. In each panel, the best-fit model (red thin solid line) is composed of a continuum (red dotted line), and a broadened $\mathrm{Fe}$ II emission complex and an $\mathrm{H} \beta$ emission line (red dashed lines). Note the extreme weakness of the $\mathrm{H} \beta$ emission line in each WLQ.

(A color version of this figure is available in the online journal.)

embedded in random noise at the observed signal-to-noise level $(\sim 10)$ and looking for the weakest such feature that would have been detected in our spectra (see, e.g., Shemmer et al. 2004).

In both sources, we detect unusually weak broad $\mathrm{H} \beta$ lines as well as prominent Fe II emission (cf. Netzer \& Trakhtenbrot 2007). Since [O III] lines are undetected, we consider the centroids of the best-fit $\mathrm{H} \beta$ lines for determining the systemic redshifts of the sources (Table 1). These redshifts are larger by $\Delta z=0.07$ than those determined from the SDSS spectra (DS09). This indicates that the SDSS redshift determination may be biased toward somewhat lower values, since the onset of the Ly $\alpha$ forest is not easily detectable at $z \sim 3.5$. The restframe spectra in Figure 1 were corrected by $z_{\text {sys }}$. We note that the broadening of the $\mathrm{Fe}$ II templates required Gaussian profiles that are significantly narrower than those of their corresponding $\mathrm{H} \beta$ lines (Table 2), which is not uncommon if most of the Fe II emission is considered to originate in an "intermediateline region" (e.g., Hu et al. 2008). Figure 2 also shows the average $\mathrm{H} \beta$ spectral region of 33 radio-quiet quasars (RQQs) at $2.0 \lesssim z \lesssim 3.5$ without broad absorption lines (hereafter, the comparison sample) from Shemmer et al. (2004) and Netzer et al. (2007), whose luminosities are comparable to those of our WLQs. The sources from the comparison sample all have $\operatorname{EW}(\mathrm{H} \beta)>46 \AA$, the maximum is $\operatorname{EW}(\mathrm{H} \beta)=256 \AA$ and the mean is $\operatorname{EW}(\mathrm{H} \beta)=107 \AA$. Thus, our two WLQs lie at the tail of an $\operatorname{EW}(\mathrm{H} \beta)$ distribution for high-redshift quasars, although this result is not as significant as the deviation of WLQs from the lognormal distribution of $\mathrm{EW}(\mathrm{Ly} \alpha+\mathrm{N} v)$ in high-redshift quasars (Section 1).

\subsection{The X-ray Spectrum of SDSS J1141+0219}

We obtained X-ray imaging spectroscopy of SDSS J1141+0219 with XMM-Newton (Jansen et al. 2001) on 2008 June 27 (data set ID 0551750301). The data were processed using standard XMM-Newton Science Analysis System ${ }^{13}$ v8.0.0 tasks. The event files were filtered in time to remove periods of flaring activity in which the count rates of each MOS (pn) detector exceeded 0.35 (1.0) counts $\mathrm{s}^{-1}$ for events having $E>10 \mathrm{keV}$; the net exposure times were $19.7 \mathrm{ks}$ and $16.7 \mathrm{ks}$ for the MOS1/MOS2 and pn detectors, respectively. For each detector, source counts were extracted using a circular aperture with $r=30^{\prime \prime}$ centered on the source and background counts were extracted from a nearby source-free region that was at least as large as the corresponding source region. The spectrum from each detector was grouped with a minimum of 20 counts per bin. The net source counts in the $0.2-10.0 \mathrm{keV}$ band were 81,128 , and 333 for the MOS1, MOS2, and pn detectors, respectively.

Joint spectral fitting of the data from all three detectors was performed with XSPEC v11.3.2 (Arnaud 1996), considering only observed frame energies above $0.44 \mathrm{keV}$. This energy range corresponds to rest-frame energies above $2 \mathrm{keV}$, where the underlying power-law hard-X-ray spectrum is less prone to contamination due to any potential absorption or soft excess emission. We fitted the spectrum with a power-law model and a Galactic-absorption component, which was kept fixed during the fit with a column density of $N_{\mathrm{H}}=2.30 \times 10^{20} \mathrm{~cm}^{-2}$ obtained from Dickey \& Lockman (1990). The power-law model consisted of a single photon index for all three detectors, while the power-law normalizations were free to vary. The best-fit photon index is $\Gamma=1.91_{-0.22}^{+0.24}\left(\chi^{2}=45.2\right.$ for 48 degrees of freedom; at $90 \%$ confidence). The X-ray spectrum and its bestfit model and residuals appear in Figure 3. Based on an $F$-test, we find that adding an intrinsic, neutral-absorption component to the above model does not improve the fit significantly and

\footnotetext{
13 http://xmm.esac.esa.int/sas
} 


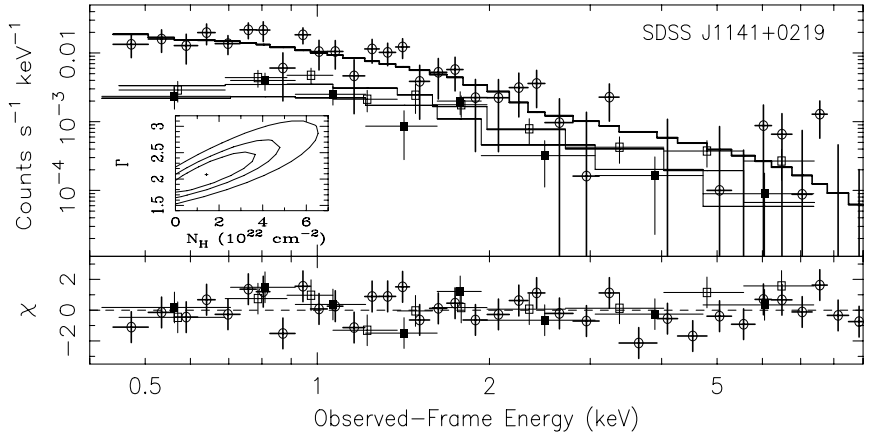

Figure 3. XMM-Newton spectrum of SDSS J1141+0219. Open circles, filled squares, and open squares represent the EPIC pn, MOS1, and MOS2 detector data, respectively. Solid lines represent the best-fit model for each spectrum and the thick lines mark the best-fit model for the pn data. The data were fitted with a Galactic-absorbed power-law model at observed frame energies $>0.44 \mathrm{keV}$. The $\chi$ residuals are in units of $\sigma$ with error bars of size 1 . The inset shows $68 \%$, $90 \%$, and $99 \%$ confidence contours for the photon index $(\Gamma)$ and an intrinsic, neutral-absorption column density $\left(N_{\mathrm{H}}\right)$, when the latter component is added to the Galactic-absorbed power-law model.

hence is not warranted by our data. The upper limit on the column density of such intrinsic neutral-absorption component is $N_{\mathrm{H}} \leqslant 3.59 \times 10^{22} \mathrm{~cm}^{-2}$ (at $90 \%$ confidence). Finally, we do not detect X-ray variability between the XMM-Newton and shallow Chandra observations of this source reported in S09.

\section{EXTREMELY HIGH ACCRETION RATES OR ANEMIC BROAD-LINE REGIONS?}

We find that at least two WLQs exhibit extremely weak UV as well as $\mathrm{H} \beta$ emission lines that place them at the tail of the corresponding EW distributions in type $1 \mathrm{AGN}$. On the other hand, a wealth of multiwavelength observations of a subset of WLQs have shown that, unlike their optical-UV emission-line properties, their broadband continua are consistent with those observed in the general quasar population at the corresponding redshifts and luminosities (e.g., DS09, S09). Such observations already question the leading hypothesis that WLQs are quasars with extremely high accretion rates (i.e., $L / L_{\text {Edd }} \gtrsim 1$ ), which has been motivated mainly by the peculiar properties of PHL 1811 (Leighly et al. 2007a, 2007b; Section 1). This quasar shows unusually weak UV emission lines and it has $L / L_{\text {Edd }} \sim 1.3$, derived from an $\mathrm{H} \beta$-based, virial determination of $M_{\mathrm{BH}}$. The properties of PHL 1811 can also be viewed as an extreme case of the anticorrelation between UV-line (e.g., C IV) strength and $L / L_{\text {Edd }}$ (Baskin \& Laor 2004). Based on the very small C IV EWs (or EW upper limits) of WLQs (DS09), and assuming this anticorrelation extends to their relatively high luminosities, the implication is $L / L_{\text {Edd }} \gtrsim 1$ for all WLQs. Such a range of $L / L_{\text {Edd }}$ constitutes the high-end tail of the $L / L_{\text {Edd }}$ distribution in $\sim 60,000$ SDSS quasars (Shen et al. 2008), and this may be analogous to the excess of WLQs with respect to the lognormal distribution of UV-line EWs in high-redshift quasars (Section 1).

We determine virial $M_{\mathrm{BH}}$ and corresponding $L / L_{\text {Edd }}$ values for our WLQs from their derived optical properties (Tables 1 and 2), utilizing the empirical BELR size-luminosity relation of Kaspi et al. (2005) modified by Bentz et al. (2009). This results in the following expressions for $M_{\mathrm{BH}}$ and $L / L_{\mathrm{Edd}}$ :

$$
\frac{M_{\mathrm{BH}}}{10^{6} M_{\odot}}=5.05\left[\frac{v L_{v}(5100 \AA)}{10^{44} \mathrm{erg} \mathrm{s}^{-1}}\right]^{0.5}\left[\frac{\operatorname{FWHM}(\mathrm{H} \beta)}{10^{3} \mathrm{~km} \mathrm{~s}^{-1}}\right]^{2},
$$

$$
L / L_{\mathrm{Edd}}=0.13 f(L)\left[\frac{v L_{v}(5100 \AA)}{10^{44} \mathrm{erg} \mathrm{s}^{-1}}\right]^{0.5}\left[\frac{\mathrm{FWHM}(\mathrm{H} \beta)}{10^{3} \mathrm{~km} \mathrm{~s}^{-1}}\right]^{-2},
$$

where we have employed Equation (21) of Marconi et al. (2004) to obtain $f(L)$, the luminosity-dependent bolometric correction to $v L_{v}(5100 \AA)$, which is 5.7 for both sources. The resulting $M_{\mathrm{BH}}$ and $L / L_{\text {Edd }}$ values (Table 2 ) are well within the ranges observed in recent quasar surveys (e.g., Shen et al. 2008), and there are no indications for extremely high accretion rates in our WLQs. In other words, although the $M_{\mathrm{BH}}$ uncertainty associated with a virial $M_{\mathrm{BH}}$ determination is typically on the order of a factor of $\sim 2$, given the luminosities of our WLQs, $L / L_{\mathrm{Edd}}>1$ would imply $\operatorname{FWHM}(\mathrm{H} \beta)<3500 \mathrm{~km} \mathrm{~s}^{-1}$ (according to Equation (2)), which is significantly lower than observed. The weakness of the $\mathrm{H} \beta$ lines in our WLQs, resulting in unusually high $I(\mathrm{Fe} \mathrm{II}) / I(\mathrm{H} \beta)$ ratios in these sources (thus implying extremely high $L / L_{\text {Edd }}$ values; Table 2 ), may appear inconsistent with the rather "normal" $L / L_{\text {Edd }}$ values we derive (e.g., Netzer et al. 2004). However, we note that several sources, mainly at $z \lesssim 0.75$, are known to have high $I(\mathrm{Fe} \mathrm{II}) / I(\mathrm{H} \beta)$ ratios and yet have $L / L_{\text {Edd }} \sim 0.1$ (e.g., Netzer \& Trakhtenbrot 2007). It is therefore possible that our WLQs constitute high-redshift outliers from the empirical $\mathrm{EW}(\mathrm{H} \beta)-L / L_{\mathrm{Edd}}$ anticorrelation of Netzer et al. (2004). We note that the absence of [O III] emission lines in our WLQs is consistent with their high $I(\mathrm{Fe} I \mathrm{I}) / I(\mathrm{H} \beta)$ ratios; furthermore, $\sim 20 \%$ of high-redshift quasars have [O III] lines that are as weak as those we measure (Netzer et al. 2004).

Another accretion rate indicator in luminous quasars is the slope of the hard-X-ray spectrum (e.g., Shemmer et al. 2008; Risaliti et al. 2009). For SDSS J1141+0219, we measure a hard$\mathrm{X}$-ray photon index of $\Gamma=1.91_{-0.22}^{+0.24}$, which is typical of luminous, high-redshift RQQs. This measurement corresponds to $L / L_{\text {Edd }} \sim 0.6$, based on the empirical $\Gamma-L / L_{\text {Edd }}$ correlation (Shemmer et al. 2008), corrected for the BELR size-luminosity relation used here. PHL 1811, for comparison, has $\Gamma=2.28_{-0.11}^{+0.12}$ (Leighly et al. 2007a), consistent within the errors, with the prediction from this correlation given its $L / L_{\text {Edd }} \sim$ 1.3. However, a more pronounced difference between PHL 1811 and SDSS J1141+0219 lies in their optical-X-ray SEDs, since the former has been found to be $\approx 30-100$ times X-ray weaker than expected from its optical luminosity (corresponding to $\approx 3 \sigma-5 \sigma$ deviation; Steffen et al. 2006; Gibson et al. 2008), while the latter is X-ray brighter than expected by a factor of $\sim 3$ (corresponding to $\sim 1.3 \sigma$ deviation; see S09). The extreme X-ray weakness of PHL 1811 has been attributed to its extremely high $L / L_{\text {Edd }}$ (Leighly et al. 2007b). We find no X-ray indication for unusually high $L / L_{\text {Edd }}$ in SDSS J1141+0219, consistent with the virial $M_{\mathrm{BH}}$ determination. This result is consistent with the S09 findings in which there is no evidence of an exceptionally high photon index in a joint X-ray spectral fit of 11 WLQs.

Based on the optical-UV spectra of two WLQs, we find that the weakness of their emission lines is not a consequence of unusual continuum-source properties. Alternatively, our results suggest that the weakness of both low- and high-ionization potential BELR lines in our two WLQs may be a consequence of unusual BELR physical properties. For example, weak (or "anemic") BELR lines across the spectrum may be due to gas deficit in the BELR manifested by a low BELR covering factor. We note that "anemic" emission-line regions in AGNs have also 
been suggested to be a result of exceptionally low $L / L_{\text {Edd }}$ (e.g., Nicastro 2000), but this scenario does not apply to WLQs that are significantly more luminous than the so-called opticallydull AGN found mainly in the local universe (e.g., Trump et al. 2009). The key to understanding the BELR in WLQs is to investigate the relationship between their high- and lowionization BELR lines (e.g., $\mathrm{C}$ IV and $\mathrm{H} \beta$ ) by additional NIR spectroscopy (to measure low-ionization lines) coupled with detailed photoionization modeling.

\section{SUMMARY}

We present new $K$-band spectra of two WLQs at $z \sim 3.5$ and an XMM-Newton spectrum of one of them. The $K$-band spectra allowed measurement of the spectral properties of the $\mathrm{H} \beta$ lines and the Fe II emission complex, and the placement of tight upper limits on the strengths of the [O III] lines. The broad $\mathrm{H} \beta$ lines in both sources are significantly weaker than those observed in typical quasars with similar luminosities and redshifts, but they still enable reliable determinations of $M_{\mathrm{BH}}$ and $L / L_{\text {Edd }}$. The results of our $K$-band and X-ray spectra do not support the idea that the weakness of the high- as well as low-ionization BELR lines in our two WLQs can be accounted for by extremely high accretion rates. Instead, our results are suggestive of "anemic" BELRs in WLQs. Clearly, high-quality NIR and X-ray spectroscopy of additional WLQs are required for robust $L / L_{\text {Edd }}$ determinations in order to test this possibility further in a much larger sample.

We gratefully acknowledge the financial support of NASA grants NNX09AF04G (O.S.) and NNX10AC99G (W.N.B.), and NSF grant AST-0707266 (M.A.S). We thank D. Chelouche, B. Wills, and M. Gaskell for fruitful discussions. This work is based partly on observations obtained at the Gemini Observatory, which is operated by the Association of Universities for Research in Astronomy, Inc., under a cooperative agreement with the NSF on behalf of the Gemini partnership: the National Science Foundation (United States), the Science and Technology Facilities Council (United Kingdom), the National Research Council (Canada), CONICYT (Chile), the Australian Research Council (Australia), Ministério da Ciência e Tecnologia (Brazil) and Ministerio de Ciencia, Tecnología e Innovación Productiva (Argentina), and partly on observations obtained with XMMNewton, an ESA science mission with instruments and contributions directly funded by ESA Member States and NASA. Funding for the SDSS and SDSS-II has been provided by the Alfred P. Sloan Foundation, the Participating Institutions, the National Science Foundation, the U.S. Department of Energy, the National Aeronautics and Space Administration, the Japanese Monbukagakusho, the Max Planck Society, and the Higher Ed- ucation Funding Council for England. The SDSS Web site is http://www.sdss.org/.

\section{REFERENCES}

Anderson, S. F., et al. 2001, AJ, 122, 503

Arnaud, K. A. 1996, in ASP Conf. Ser. 101, Astronomical Data Analysis Software and Systems V, ed. G. H. Jacoby \& J. Barnes (San Francisco, CA: ASP), 17

Baskin, A., \& Laor, A. 2004, MNRAS, 350, L31

Bentz, M. C., Peterson, B. M., Netzer, H., Pogge, R. W., \& Vestergaard, M. 2009, ApJ, 697, 160

Boroson, T. A., \& Green, R. F. 1992, ApJS, 80, 109

Collinge, M. J., et al. 2005, AJ, 129, 2542

Diamond-Stanic, A. M., et al. 2009, ApJ, 699, 782 (DS09)

Dickey, J. M., \& Lockman, F. J. 1990, ARA\&A, 28, 215

Fan, X., et al. 1999, ApJ, 526, L57

Gibson, R. R., Brandt, W. N., \& Schneider, D. P. 2008, ApJ, 685, 773

Hodapp, K. W., et al. 2003, PASP, 115, 1388

Hryniewicz, K., Czerny, B., Nikołajuk, M., \& Kuraszkiewicz, J. 2010, MNRAS, 404, 2028

Hu, C., Wang, J.-M., Ho, L. C., Chen, Y.-M., Bian, W.-H., \& Xue, S.-J. 2008, ApJ, 683, L115

Jansen, F., et al. 2001, A\&A, 365, L1

Kaspi, S., Maoz, D., Netzer, H., Peterson, B. M., Vestergaard, M., \& Jannuzi, B. T. 2005, ApJ, 629, 61

Leighly, K. M., Halpern, J. P., Jenkins, E. B., \& Casebeer, D. 2007a, ApJS, 173,

Leighly, K. M., Halpern, J. P., Jenkins, E. B., Grupe, D., Choi, J., \& Prescott, K. B. 2007b, ApJ, 663, 103

Marconi, A., Risaliti, G., Gilli, R., Hunt, L. K., Maiolino, R., \& Salvati, M. 2004, MNRAS, 351, 169

McDowell, J. C., Canizares, C., Elvis, M., Lawrence, A., Markoff, S., Mathur, S., \& Wilkes, B. J. 1995, ApJ, 450, 585

Netzer, H., Lira, P., Trakhtenbrot, B., Shemmer, O., \& Cury, I. 2007, ApJ, 671, 1256

Netzer, H., Shemmer, O., Maiolino, R., Oliva, E., Croom, S., Corbett, E., \& di Fabrizio, L. 2004, ApJ, 614, 558

Netzer, H., \& Trakhtenbrot, B. 2007, ApJ, 654, 754

Nicastro, F. 2000, ApJ, 530, L65

Plotkin, R. M., et al. 2010, AJ, 139, 390

Risaliti, G., Young, M., \& Elvis, M. 2009, ApJ, 700, L6

Schneider, D. P., et al. 2005, AJ, 130, 367

Shemmer, O., Brandt, W. N., Anderson, S. F., Diamond-Stanic, A. M., Fan, X., Richards, G. T., Schneider, D. P., \& Strauss, M. A. 2009, ApJ, 696, 580 (S09)

Shemmer, O., Brandt, W. N., Netzer, H., Maiolino, R., \& Kaspi, S. 2008, ApJ, 682,81

Shemmer, O., Netzer, H., Maiolino, R., Oliva, E., Croom, S., Corbett, E., \& di Fabrizio, L. 2004, ApJ, 614, 547

Shemmer, O., et al. 2006, ApJ, 644, 86

Shen, Y., Greene, J. E., Strauss, M. A., Richards, G. T., \& Schneider, D. P. 2008, ApJ, 680, 169

Skrutskie, M. F., et al. 2006, AJ, 131, 1163

Steffen, A. T., Strateva, I., Brandt, W. N., Alexander, D. M., Koekemoer, A. M., Lehmer, B. D., Schneider, D. P., \& Vignali, C. 2006, AJ, 131, 2826

Stocke, J. T., \& Perrenod, S. C. 1981, ApJ, 245, 375

Trump, J. R., et al. 2009, ApJ, 706, 797

Vanden Berk, D. E., et al. 2001, AJ, 122, 549

Vasudevan, R. V., \& Fabian, A. C. 2007, MNRAS, 381, 1235

York, D. G., et al. 2000, AJ, 120, 1579 